\title{
Hydraulic logic gates: building a digital water computer
}

\author{
Nicolas Taberlet, ${ }^{1,}$ a) Quentin Marsal, ${ }^{1}$ Jérémy Ferrand, ${ }^{1}$ and Nicolas Plihon ${ }^{1}$ \\ Univ Lyon, Ens de Lyon, Univ Claude Bernard, CNRS, Laboratoire de Physique, Département de Physique, \\ F-69342 Lyon, France
}

(Dated: 13 December 2019)

In this article, we propose an easy-to-build hydraulic machine which serves as a digital binary computer. We first explain how an elementary adder can be built from test tubes and pipes (a cup filled with water representing a 1, and empty cup a 0). Using a siphon and a slow drain, the proposed setup combines AND and XOR logical gates in a single device which can add two binary digits. We then show how these elementary units can be combined to construct a full 4-bit adder. The sequencing of the computation is discussed and a water clock can be incorporated so that the machine can run without any exterior intervention.

\section{INTRODUCTION}

Physics has been the cornerstone of all major technical advances throughout the history of computing ${ }^{1}$, with fundamental concepts emerging from solid and fluid mechanics, electronics and solid-state physics, as well as quantum physics in recent years. In mechanics, pin-wheel computers include Pascal's calculator ${ }^{2}$ (designed as early as 1642, the Pascaline was the first calculator to be patented), the arithmometer (the first mass-marketed commercial calculator, about 5500 units were produced and sold world-wide $)^{3}$, Babbage's programmable analytical engine (designed in 1834 and considered to be the ancestor of modern computers ${ }^{4}$ ) or the famous cypher enigma machine, most notably used by Nazi Germany during WWII ${ }^{5}$. In fluid mechanics, processing and computing devices include the Tesla valve $^{6,7}$, pressure-actuated valves acting as analog transistors, non-linear amplifiers relying on deflecting a weaker jet striking a stronger jet of fluid $^{8}$, digital pneumatic logic gates $^{9,10}$, as well as the MONIAC (a water-based analog computer designed to model economy). ${ }^{11,12}$ It is important to note that computing using advanced fluidics systems was deeply studied and implemented in operational systems until the 1970's ${ }^{13}$ ? , before fluidics could not compete against electronics. In electronics, vacuum tubes ${ }^{14}$ remained fundamental components for electronics throughout the first half of the $\mathrm{XX}^{\text {th }}$ century ${ }^{15}$ until the invention of semiconductors and the discovery of the transistor effect (which was honored with the Nobel Prize in Physics in $1956)^{16}$. Another leap came with the invention of the integrated circuit (patented in 1959) for which Kilby won the 2000 Nobel Prize in Physics ${ }^{16}$. Finally, the concept of quantum computation has attracted much interest in the past two decades ${ }^{17-19}$. While promising recent technical developments in this field brought the concept closer to reality ${ }^{20,21}$, building a fully-functioning large-scale quantum computer still remains a challenge. ${ }^{22}$

\footnotetext{
a) Electronic mail: nicolas.taberlet@ens-lyon.fr
}

One of the most fundamental tasks any computer has to perform is arithmetic operations. Adding binary numbers $(a$ and $b)$ is nothing more than the routine children are taught in elementary school. For example, $1101+$ 1001 (i.e. 13+9) is computed as follows (starting from the right-most digit):

- 1 and 1 is zero and 1 is carried

- 0 and 1 is zero, which makes 1 with the carry

- 1 and 0 is one,

- 1 and 1 is zero and 1 is carried

- resulting in $10110(=22)$.

In this paper we present an easy-to-built hydraulic digital adding machine with relies on simple physical principles proposed by Gitton ${ }^{23}$. We first explain (section II) how using a siphon, a slow drain and a overflowing

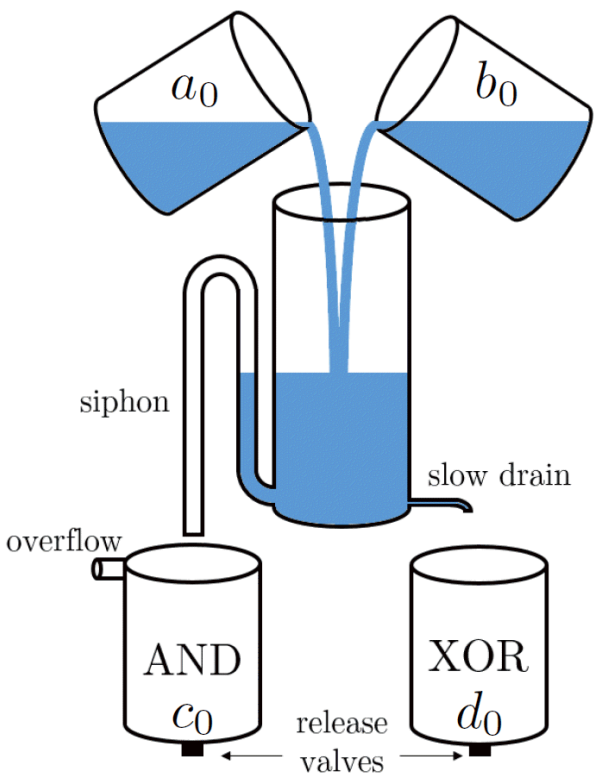

FIG. 1. Hydraulic digital arithmetic unit: the content of one (and only one) cup (i.e. 1+0) slowly leaks out of the central container through the XOR gate while the content of two cups $(1+1)$ primes the siphon and is rapidly evacuated through the AND gate. The smaller cups have a volume of $25 \mathrm{~mL}$ while that of the central container is $50 \mathrm{~mL}$, and the height of the siphon is $2 / 3$ of the total height. 
gauge one is able to construct AND and XOR logic gates in one single device. In section III we explain how these processing units can be arranged so as to form a full binary adding machine, while section IV describes the experimental 4-bit water computer that we have built.

\section{ADDING TWO DIGITS}

\section{Logical operations}

Adding the first digit of each number $\left(a_{0}\right.$ and $\left.b_{0}\right)$ yields the first digit of their sum $\left(d_{0}\right)$ and the first carry $\left(c_{0}\right)$. The carry is 1 if and only if $a_{0}=b_{0}=1$ while the first digit of the sum is 1 if and only if either $a_{0}$ or $b_{0}$ is 1 (not both) (see Table I). In terms of logical operations this can be summarized as:

$$
\begin{gathered}
\left\{\begin{array}{l}
c_{0}=a_{0} \text { AND } b_{0} \\
d_{0}=a_{0} \text { XOR } c_{0}
\end{array}\right. \\
\begin{array}{|l|l|l|l|l|}
\hline a_{0} & 0 & 0 & 1 & 1 \\
b_{0} & 0 & 1 & 0 & 1 \\
\hline c_{0} & 0 & 0 & 0 & 1 \\
d_{0} & 0 & 1 & 1 & 0 \\
\hline
\end{array}
\end{gathered}
$$

TABLE I. Carry, $c_{0}$, and digit, $d_{0}$, of the sum of $a_{0}$ and $b_{0}$. In logical operations: $c_{0}=a_{0}$ AND $b_{0}$ while $d_{0}=a_{0}$ XOR $b_{0}$.

\section{Hydraulic AND and XOR gates}

The addition of the first two digits therefore only requires an AND and XOR gate. The hydraulic device shown in Fig. 1 combines both gates in one single apparatus. It consists in a central container into which the content of two smaller cups (representing $a_{0}$ and $b_{0}$ ) is simultaneously poured. The cups can be either completely empty (representing a 0) or completely full (representing a 1) and their volume is half that of the central container. A small hole is drilled at the bottom of the main container (bottom right in Fig. 1) so that its content can slowly drain out. On the other side (bottom left in Fig. 1), a large hole is connected to a siphon, whose height is greater than half of that of the main container.

If one and only one of the top cups is full, the water poured into the central container gradually winds up in the bottom-right cup. On the other hand, if both top cups are full, the siphon is almost instantly primed and the content of the main container quickly empties out into the bottom left cup, whose overflow tube ensures that the results remains binary. Overflow is a generic feature of fluidics devices in which vents were widely implemented ${ }^{24}$. Note that even when the siphon is primed and the water is quickly evacuated, a small amount still leaks through the slow drain. The diameters of the drain and siphon must be chosen so that the flow rate through the siphon is far greater.

Therefore, the bottom cups give the results of the logical operation AND (for the left cup) and XOR (for the right cup). The smaller cups (top and bottom) act as memory units while the larger central container incorporates the logic gates.

It is important to note that the gates presented here are basic digital components which do not rely on basic analog hydraulic components. This is a fundamental difference with electronic digital circuits, designed as the association of several analog circuits, namely transistors ${ }^{25}$. Technical details

The central container is a $50 \mathrm{~mL}$ plastic test tube, 27 $\mathrm{mm}$ in diameter. The smaller cups (memory units) are made of the same test tube cut in half $(25 \mathrm{~mL})$. The hole through which water slowly drains out is pierced using an incandescent needle which gives a diameter of typically $500 \mu \mathrm{m}( \pm 20 \%)$. The siphon and the overflow tube are built using plastic straws ( $5 \mathrm{~mm}$ in diameter). Straws are very convenient as they are made to be bent without buckling or pinching. In this device it took approximatively 2 min for the content of one cup $(25 \mathrm{~mL})$ to slowly drain through the XOR gate whereas it takes less than 5 $\mathrm{s}$ for the content of two cups $(50 \mathrm{~mL})$ to quickly evacuate through the AND gate (the primed siphon).

In order to guide the water gradually leaking through the XOR gate, a straw (see Fig. 3) is glued around the small hole. A release valve, consisting in a $5 \mathrm{~mm}$ rubber stopper, is placed at the bottom of each memory unit for further processing.

Note that capillary effects may cause a number of problems: some liquid can stay trapped in the straws while the flow through the small drain can be hindered. This can be overcome by reducing the surface tension of the liquid. Soap must be avoided since it creates bubbles or foam but adding a small amount of ethanol to the water is enough to considerably help.

\section{FULL-ADDER}

\section{Adding the $n^{\text {th }}$ digits}

The device sketched in Fig. 1 allows one to add two 1-bit numbers and yields a 2-bit number and is known as a half-adder. ${ }^{26}$ As explained above, it can be used to compute the sum of the first digits $\left(a_{0}\right.$ and $\left.b_{0}\right)$. However, when adding any $n^{\text {th }}$ digits $\left(a_{n}\right.$ and $\left.b_{n}\right)$, the carry $\left(c_{n-1}\right)$ originating from all previous calculations must be taken into account. A full-adder is therefore an operation with three inputs and two outputs : $\left(a_{n}, b_{n}, c_{n-1}\right) \rightarrow\left(c_{n}, d_{n}\right)$.

As illustrated in the introductory addition of 1101 (13) and 1001 (9), a full-adder must first add the $n^{\text {th }}$ digits together, their XOR result then needing to be added to the $n-1^{\text {th }}$ carry. That second operation gives the $n^{\text {th }}$ digit of the sum $\left(d_{n}\right)$. The AND results of both computations give the $n^{\text {th }}$ carry. Note that not possible for both AND gates to yield 1 and no further precaution (such as an additional OR gate) needs to be taken. The combination of two half-adders is known as a full-adder and a sketch of a device built with our hydraulic gates 
is shown in Fig. 2.

Clock rate

When using a full-adder, great care must be given to the timing of the operations. The first half-adder (container 1 in Fig. 2) can perform its computation as soon as the device is started $(t=0)$ but the second computation (container 2 in Fig. 2) must wait for all previous operations to have been performed. As explained above, the output of a AND gate in our device is given in less than $5 \mathrm{~s}$ while that of the XOR gate is given in $T=2 \mathrm{~min}$. Therefore, one must ensure that enough time is given for all halfadditions to be over before further processing. Therefore, the second operation of the $n^{t h}$ full adder must be performed at time $t=n T$. Our water computer can only boast a disheartening clock rate of the order of $0.01 \mathrm{~Hz}$, far less than the typical $10^{10} \mathrm{~Hz}$ of modern microchips. ${ }^{27}$ For the sake of simplicity, the version of the full adder presented here does not include a fully integrated hydraulic clock and relies on the successive manual opening of valves. However, it is in principle possible to build a dedicated clock which uses hydraulically controlled valves and whose sequencing is governed by the associated timing diagrams of the computer ${ }^{24}$.

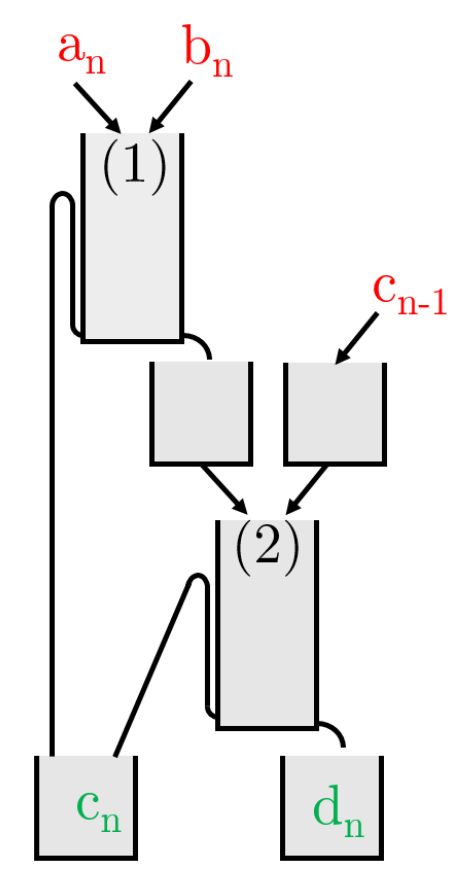

FIG. 2. Sketch of a full-adder allowing one to add two digits $\left(a_{n}\right.$ and $\left.b_{n}\right)$ while taking into account the carry $\left(c_{n-1}\right)$ resulting from the earlier stages of computation. The full-adder yields the $n_{t h}$ digit of the sum $\left(d_{n}\right)$ as well a the $n_{t h}$ carry $\left(c_{n}\right)$. The computation must be sequenced so as to ensure that the second computation of the stage (2) is triggered only after all previous operations have terminated.

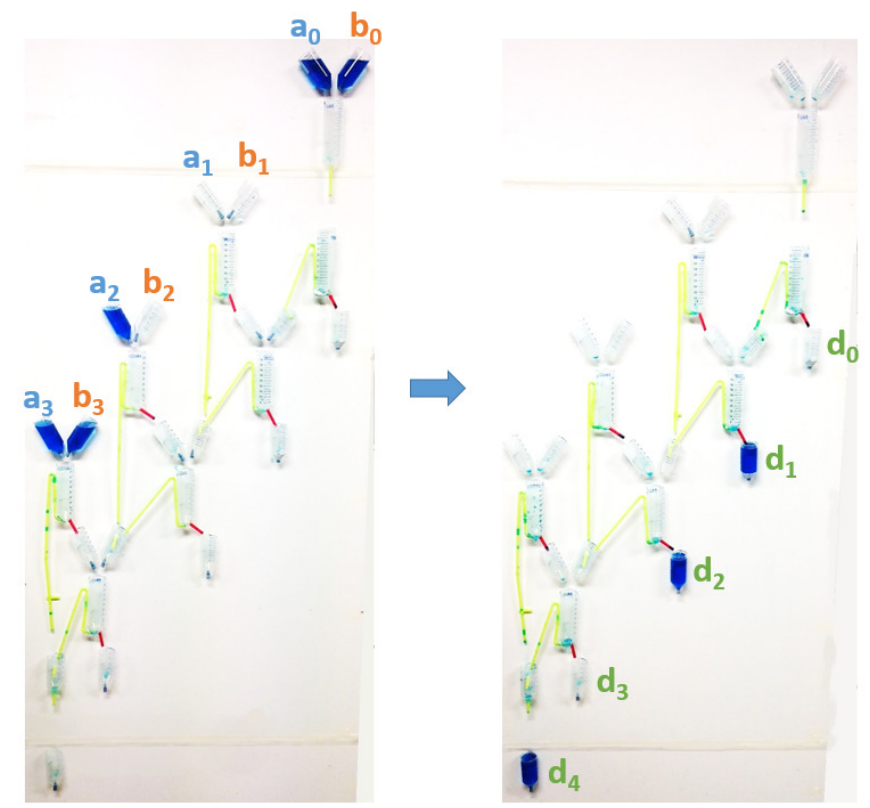

FIG. 3. Pictures of our 4-bit hydraulic digital adder made of 7 processing units. The initial state shows the two numbers to be added $(a=1101)$ and $(b=1001)$ and the result can be read in the final state $(d=10110)$ after 8 minutes of computation.

\section{4-BIT HYDRAULIC DIGITAL COMPUTER}

\section{A full 4-bit adder}

Figure 3 shows a 4-bit adding machine built by assembling 7 half-adders. As explained in section II, the memory units (the smaller cups) are emptied out into the processing units (the central containers) through 5 -mm holes at their bottom when rubber stoppers are removed. The red straws channel the results of the XOR gates into the memory units while the AND gates (the siphons) are made of green straws. For clarity, the water is died blue. The initial state is shown on Fig. 3a: $a=1101(=13)$ and $b=1001(=9)$. After 8 minutes, the computation is complete and the result can be read in the bottom memory units (Fig. 3b). As expected the sum is indeed $d=10110(=22)$.

\section{Subtraction}

The digital hydraulic adder presented above can also be used to perform subtractions (13-9 for instance) using a method known as the two's complement ${ }^{28}$, that allows one to use signed binary numbers. The two's complement defines negative 9 as $-9=\operatorname{NOT}(9)+1$, to which 13 can be added. Table II presents the operation. The result must not be interpreted as a 5-digit number since obviously the difference between two 4-digit numbers is less than 15. Instead, the $5^{t h}$ is only an indication of the sign. In the example shown in Table II, the result, 1 0100, must be read as +4 .

The NOT operation can be performed using the hydraulic half-adder presented in section II since for each 


\begin{tabular}{|c|lllll|}
\hline 9 & 1 & 0 & 0 & 1 \\
$\operatorname{NOT}(9)$ & & 0 & 1 & 1 & 0 \\
$\operatorname{NOT}(9)+1$ & & 0 & 1 & 1 & 1 \\
+13 & & 1 & 1 & 0 & 1 \\
\hline$-9+13$ & 1 & 0 & 1 & 0 & 0 \\
\hline
\end{tabular}

TABLE II. Computation of 13-9 using the two's complement method in which -9 is given by NOT $(9)+1$. Since the difference cannot exceed $2^{4}-1=15$, the $5^{\text {th }}$ digit must be ignored, and the result is $0100=4$, as expected.

digit $\operatorname{NOT}\left(a_{n}\right)=a_{n}$ XOR 1 . One simply needs to collect the result of the XOR gate (the slow drain), the $b$ cup being always filled, while the result of the AND gate is irrelevant. The 4-bit adder presented in section IV can then first be used to add 1 to -9 , and then used again to add 13 to the result.

\section{v. CONCLUSION}

In this paper, the principle of a digital binary adder was described and the use of the AND and XOR logic gates was explained. We proposed a simple arithmetic unit relying on hydraulic principles which combines both AND and XOR gates in a single device, easily built with plastic test tubes and straws. Arranging 7 of these elementary processing units, a functioning hydraulic 4-bit digital adder was constructed. The proposed design can be improved in several ways.

A water-based clocked can be built to automatically trigger the computation every two minutes. Weights attached to the two rubber stoppers above the processing unit, through initially loose strings, can be placed on a balance beam with a leaking cup of water acting as a counterweight. After the desired duration, the balance beam tips over, causing the weight to fall and to pull the stoppers out, thereby releasing the water, i.e. triggering the computation. Such a simple independent clock allows the machine to run free of any human intervention during the computing process (which viewers may perceive as a deception).

Moreover, it is well known that during the adding process information is lost (in mathematical terms, addition is not bijective). Obviously, for instance adding 13 and 9 yields the same results as adding 8 and 14. However, in our 4-bit adding machine, a trick might be used to identify the original numbers ( $a$ and $b$ ) from the result $(d)$. If each initial digit is assigned a specific color, then analyzing the hue of the final digits in the sum could allow one to deduce the value of the two initial numbers.

\section{ACKNOWLEDGMENTS}

The authors acknowledge support from the University Lyon Claude Bernard, the Société Française de Physique and from the École Normale Supérieure de Lyon and its Physics Department and Laboratoire de Physique.

${ }^{1}$ M. Wolf, The Physics of Computing. Elsevier, 2016.

${ }^{2}$ D. Adamson, Blaise Pascal: mathematician, physicist and thinker about God. Springer, 1994.

${ }^{3} \mathrm{E}$. D. Reilly, Milestones in computer science and information technology. Greenwood Publishing Group, 2003.

${ }^{4}$ B. V. Bowden, "Faster than thought: a symposium on digital computing machines," 1953.

${ }^{5}$ W. Kozaczuk, Enigma: how the German machine cipher was broken, and how it was read by the Allies in World War Two. Foreign intelligence book series, University Publications of America, 1984.

${ }^{6} \mathrm{P}$. Tabeling, Introduction to microfluidics. Oxford University Press on Demand, 2005.

${ }^{7}$ D. E. Angelescu, Highly integrated microfluidics design. Artech House, 2011.

${ }^{8}$ T. M. Hunt, T. Hunt, N. Vaughan, and N. Vaughan, The hydraulic handbook. Elsevier, 1996.

${ }^{9}$ M. Rhee and M. A. Burns, "Microfluidic pneumatic logic circuits and digital pneumatic microprocessors for integrated microfluidic systems," Lab on a Chip, vol. 9, no. 21, pp. 3131-3143, 2009.

${ }^{10} \mathrm{R}$. Dewhirst, Passive Pneumatic Half-adder with No Moving Parts. Massachusetts Institute of Technology, Department of Mechanical Engineering, 1964.

${ }^{11} \mathrm{C}$. Bissell, "Historical perspectives-the moniac a hydromechanical analog computer of the 1950s," IEEE Control Systems, vol. 27 , no. 1 , pp. 69-74, 2007.

${ }^{12} \mathrm{D}$. Colander, "The moniac, modeling, and macroeconomics," Economia politica, vol. 28, no. 1, pp. 63-82, 2011.

${ }^{13} \mathrm{~J}$. Kirshner, Design theory of fluidic components. Academic Press, New York, 1975.

${ }^{14} \mathrm{H}$. Schultz and W. Wadey, "A laboratory course in electronics," American Journal of Physics, vol. 19, no. 4, pp. 214-223, 1951.

${ }^{15} \mathrm{~S}$. Macdonald and E. Braun, "The transistor and attitude to change," American Journal of Physics, vol. 45, no. 11, pp. 10611065, 1977.

${ }^{16}$ N. Weste, D. Harris, and A. Banerjee, "Cmos vlsi design," A circuits and systems perspective, vol. 11, p. 739, 2005.

${ }^{17}$ C. H. Bennett and D. P. DiVincenzo, "Quantum information and computation," Nature, vol. 404, no. 6775, pp. 247-255, 2000.

${ }^{18}$ N. D. Mermin, "From cbits to qbits: Teaching computer scientists quantum mechanics," American Journal of Physics, vol. 71, no. 1, pp. 23-30, 2003.

${ }^{19}$ D. Candela, "Undergraduate computational physics projects on quantum computing," American Journal of Physics, vol. 83, no. 8, pp. $688-702,2015$.

${ }^{20}$ L. DiCarlo, J. Chow, J. Gambetta, L. S. Bishop, B. Johnson, D. Schuster, J. Majer, A. Blais, L. Frunzio, S. Girvin, et al., "Demonstration of two-qubit algorithms with a superconducting quantum processor," Nature, vol. 460, no. 7252, pp. 240-244, 2009.

${ }^{21}$ N. Somaschi, V. Giesz, L. De Santis, J. Loredo, M. P. Almeida, G. Hornecker, S. L. Portalupi, T. Grange, C. Antón, J. Demory, et al., "Near-optimal single-photon sources in the solid state," Nature Photonics, 2016.

${ }^{22}$ C. P. Williams, Explorations in quantum computing. Springer Science \& Business Media, 2010.

23 "B. gitton." https://en.wikipedia.org/wiki/Bernard_Gitton. Unfortunatly Gitton's design seems to have remained unpublished but a device using his concept was built at the Technisches Museum of Vienna, Austria.

${ }^{24}$ C. A. Belsterling, Fluidic systems design. Wiley Interscience, New York, 1971.

${ }^{25} \mathrm{P}$. Horowitz and W. Hill, The art of electronics. Cambridge Univeristy Press, 1989.

${ }^{26} \mathrm{D}$. Page, A practical introduction to computer architecture. Springer Science \& Business Media, 2009. 
${ }^{27}$ M. J. Rodwell, High-speed integrated circuit technology: towards $100 \mathrm{GHz}$ logic, vol. 21. World Scientific, 2001.

${ }^{28} \mathrm{~L}$. Null, J. Lobur, et al., The essentials of computer organization and architecture. Jones \& Bartlett Publishers, 2014. 